\title{
Masters courses in psychiatry
}

\author{
Philip J. Shoebridge and Mark McCartney
}

\begin{abstract}
The variations in structure and content between Masters degree courses offered to psychiatiy trainees around the country are documented. Most courses are integrated to some degree with statutory Membership teaching. The extent to which courses differ is likely to surprise readers. The implications for the junior trainee as well as for consultant or senior registrar appointment committees is discussed. Universities act autonomousty, hence a Masters degree awarded by one may bear no relation to that awarded by another.
\end{abstract}

In October 1994 a conference was held in Leeds for organisers of Masters degree courses in psychiatry. The differences in structure and content of courses leading to apparently equivalent academic qualifications were strikingly apparent. Two trainees (ourselves) attended and were stimulated to research the differences between Masters courses around the United Kingdom.

\section{Background}

All trainees wishing to sit the MRCPsych examination are required by the College to attend an academic course and thus all training centres have provided a 'taught membership course'. Recently more university departments of psychiatry are offering courses which aim to offer some or all trainees the opportunity to gain a further qualification, usually a Masters degree (British Council, 1993).

The provision of such Masters courses shows considerable national variation. In some areas there are, as yet, no such courses available. In others, Masters courses are only available for a few trainees, contrasting with yet others where all trainees are obliged to register as the Masters course also functions as the only available MRCPsych course.

Trainees' primary objectives in undertaking Masters courses include obtaining the MRCPsych and then a place on the senior registrar (SR) rotation of their choice. In a survey of Fellows and new Members of the College, with regard to which factors were considered important for SR short-listing, a "higher degree related to psychiatry" was rated particularly highly (Haigh \& Weaver, 1993).

The conference revealed clear differences in the prevailing culture of academic departments, particularly to what extent each encourages their career trainees to get a Masters degree, and whether they use this as a bench-mark to judge which trainees will be offered preferment to the local SR rotations or other academic opportunities. In Cardiff the expectation is that trainees will only be considered for the SR rotation if they have an MSc. In Manchester and at the Maudsley promotion occurs before the degree can be awarded but those doing the MSc are seen as a cadre of potential academics. At St George's very few trainees take Masters courses, preferment resting on other academic achievements such as publications or current involvement in research.

The Collegiate Training Committee (Royal College of Psychiatrists, 1994) have expressed concern that Masters courses should not be compulsory, taking the view that they predominantly require original research and it is debatable whether all trainees need to undertake such activities. Bowen \& Cox (1993) also emphasise how research currently seems to hold a pre-eminence in terms of career progress that is not justified in the reality of NHS consultants' practice.

Table 1 serves to illustrate the current situation, comparing the 11 centres who currently offer such courses.

\section{The overlap in the Masters and the taught membership courses}

There are striking variations in the relationship between the membership courses and the Masters course. Whereas in Cardiff, Edinburgh. The Maudsley, Leeds and Nottingham the Masters course completely subsumes the whole MRCPsych course, at the United Medical and Dental Schools (UMDS) the two are entirely separate. For 


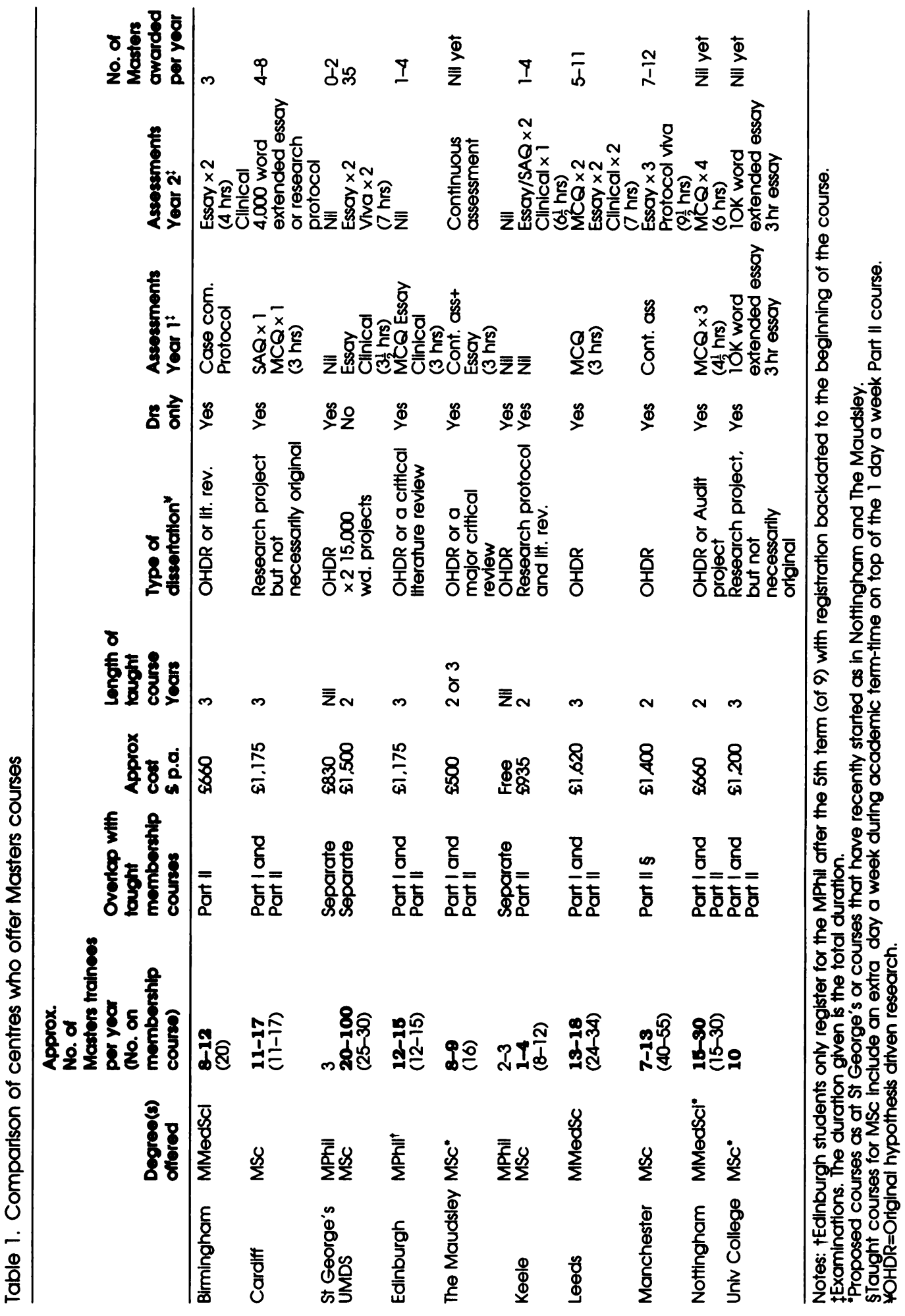


most there is overlap restricted to the Part II course. Manchester is one of the few centres where MSc trainees get an extra half day in addition to the usual full day's Part II teaching. At St George's, UMDS and for some Birmingham trainees the membership course is taught in only half a day a week.

Most courses encompass the broad remit of general and sub-speciality psychiatry because of the intimate relationship between the membership and Master's courses. In those centres where the two run independently the course material is much more diverse; thus the St George's MSc will be restricted to behaviour and cognitive psychotherapy and at Guy's, specialist option modules (two of which must be taken in addition to the basic module) include Transcultural Psychiatry and Anthropology', 'Community Mental Health', and 'Marital and Sexual Therapy'. Dissertations have included projects on spiritual healing.

\section{MPhils, MScs, MMed Scis or diplomas}

Edinburgh trainees are only offered the option of an MPhil. The Maudsley and St George's currently offer both MPhils and MScs. (The Maudsley regards its MPhil as the first step towards a PhD). Birmingham, Leeds and Nottingham offer MMedScis. The remainder are MScs. These are assumed to be technically equivalent degrees.

An advantage of the MPhil is that unlike most Masters it can be upgraded to an MD or PhD (dependent upon varying university regulations). Many of the senior academic staff at the conference were unaware of their own university's requirements in this area.

Some centres offer the option of a diploma to students who do not wish to complete the dissertation (Birmingham) or who do not get adequate grades to continue with the Masters course (Cardiff). Several more centres are planning to offer a diploma. Whether they offer a useful qualification for career trainees is doubtful (Haigh \& Weaver, 1993).

\section{Applicants}

Most Masters courses are available only to psychiatric trainees. At the UMDSand St George's other post-graduate students in the University may apply, only one third of the students on the UMDS Masters being doctors. Keele is considering marketing its MSc for GP trainees.
Generating revenue from increasing numbers of applicants may prompt organisers to widen the degree's appeal.

\section{Examinations}

Assessment of progress by formal examination is not uniform. The first two years at Leeds includes eight hours of MCQ and essay papers and two clinical exams, contrasting with Edinburgh where there is only one three hour exam at the end of the first year, before trainees even register for the MPhil.

All universities require that Masters dissertations are assessed by both internal and external examiners. Viva examination of candidates on their final dissertations or research proposals is usual in some institutions (The Maudsley and Manchester) but does not occur in others (Cardiff and Keele).

\section{Quality of dissertations}

Quantifying the differences in the nature and quality of submitted dissertations is confounded by the lack of standardisation. At Keele a literature review or an appended research protocol' constitutes a sufficient dissertation contrasting with many Masters courses where original hypothesis driven research is mandatory. Nottingham is proposing an option to undertake a substantial audit project. Some centres' research dissertations have a reputation of approaching $\mathrm{PhD}$ or $\mathrm{MD}$ standard, which begs the question as to whether these trainees are being ill-advised or are being poorly rewarded for their undertakings. There appear to be no national standards, but a locally determined culture that appears to perpetuate what is considered acceptable. External examiners are evidently guided by these local norms.

\section{Finances}

Masters fees vary from $£ 1,620$ per annum at Leeds to $£ 500$ at the Maudsley. There is a correlation between charges and the extent to which the Masters is linked to the membership courses as the latter are regulated by the academic department of psychiatry rather than by the parent university.

The attitude of the regional post-graduate dean may influence the local organisation of Masters courses as they are financed from 
their training budgets. Their priorities are to encourage the best possible academic and clinical training to equip future consultants and to ensure they are getting value for money for their trainees. Psychiatry trainees consume a disproportionate slice of their budget. Regionally, deans have between $£ 300$ and $£ 680$ per trainee which will mean the much more substantial fees for Masters courses can only be sustained by other trainees not spending their own budget allocation. There are wide variations nationally in their attitudes to Masters courses with recently expressed concerns about a general proliferation of such courses available to all medical specialities threatening to overwhelm their resources.

\section{Idiosyncrasies}

Cardiff offer all candidates video training in academic presentation skills. Leeds incorporate a clinical neurology exam and practical workshops on computing skills. Birmingham and The Maudsley have a written exam on research methodology, the Maudsley's including ethical questions raised by clinical research. At the Maudsley trainees have to contribute $£ 125$ p.a. towards their fees. At St George's all trainees on the membership course get the chance to dissect a brain. At the UMDS each Master's course peer group includes all mental health professionals and much of the teaching is in the evenings and weekends.

\section{Puture prospects and conclusions}

This clearly heterogeneous group of postgraduate courses conveniently subsumed under the term 'Masters courses' is confusing to trainees and senior psychiatrists alike. The diversity of form and content of these courses is unlikely to narrow and one might argue that it should not. Clear information should be available to trainees to enable them to gain informed and individually tailored advice regarding postgraduate qualifications (Bende \& Hopkins, 1994) and to maintain choice by the continuing availability and recognition of a diversity of possible postgraduate educational experiences.

There are currently no national criteria based standards for Masters degree courses in psychiatry (although the external examiner system aims to reduce discrepancies). A Masters in psychiatry will mean different things to different people. Trainees applying for jobs in other regions should clarify the requirements of their own Masters in order that they be appropriately credited. It remains unclear how SR and consultant appointment committees view these qualifications, and if trainees consider the qualification a substitute for academic publication.

There is a movement within universities to have modular Masters courses with credits transferable between centres, reviewed by the Higher Education Funding Council. This might help standardisation, increase the diversity of academic aspects of psychiatry available for study, and facilitate trainees moving between centres as personal or training imperatives dictate.

\section{Acknowledgements}

We wish to thank Dr David Owens, Dr Margaret Oates, Professor Shon Lewis, and Professor Channi Kumar for making constructive suggestions to earlier drafts of this article and to the many course organisers and administrators who supplied us with factual information regarding their courses.

\section{References}

Bende, B. \& Hopkins, R. (1994) Career guidance for psychiatric trainees. Psychiatric Bulletin, 18, 704-705.

BOWEN. J. \& CoX. S. (1993) Registrars with research - the right stuff, or the wrong stuff? Psychiatric Bulletin. 17. 540-541.

BRTISH CouncIL (1993) Guide to Postgraduate Degrees, Diplomas \& Courses in Medicine. Intelligene in association with the National Advice Centre for Postgraduate Medical Education.

HAIGH, R. \& WEAVER, M. (1993) Rumour, myth and reality at SR interviews. Psychiatric Bulletin, 17, 541-543.

Royal College of PSYchiatrists Colleglate Trainees COMMITTEE (1994) CTC position on Structured Training (CTC 39/94). Chairman: Dr Steffan Davies.

*Philip J. Shoebridge, Senior Registrar in Child and Adolescent Psychiatry, The Gardener Unit, Prestwich Hospital, Bury New Road, Manchester M25 3BL; and Mark McCartney, Registrar, Mid-Trent Rotation, Aston Hall Hospital, Aston on Trent DE7 1AL

*Correspondence 